www.ccsenet.org/journal.html

\title{
Existence of Nonoscillatory Solution of High Order Linear Neutral Delay Difference Equation
}

\author{
Shasha Zhang, Xiaozhu Zhong, Ping Yu, Wenxia Zhang \& Ning Li \\ Department of Mathematics \\ Yanshan University \\ Qinhuangdao 066004, China \\ E-mail:shashazm@163.com
}

Supported by the Foundation for the natural science of Hebei province of China (Z2007431)

\begin{abstract}
Consider the high order neutral delay difference equation with positive and negative coefficients $\Delta^{l+1}[x(n)+p x(n-\tau)]+R_{1}(n) x\left(n-\delta_{1}\right)-R_{2}(n) x\left(n-\delta_{2}\right)=o$

Where $p \in R ; \tau \in N(1) ; \delta_{1}, \delta_{2} \in N ;\left\{R_{1}(n)\right\},\left\{R_{2}(n)\right\}$ are positive real sequences. A sufficient condition for the existence of the eventually positive solution of the above equation is set forward in terms of $\sum_{n=1}^{+\infty} n^{l} R_{i}(n)<+\infty, i=1,2, n \in N\left(n_{0}\right)$. This result got rid of a quite strong tentative of existing literature, which improve
\end{abstract} the relevant theorem.

Keywords: Positive and negative coefficients, Neutral difference equation, Eventually positive solution

\section{Introduction}

Nowadays, as the rapid development of computer science, automation technology, biology and numerical research, neutral delay differential equations oscillation study attracts the attention of many scholars, and some research achievements have been taken in this area. At the same time, a difference equation with positive and negative coefficients attracting more attention becomes a new study field. However, only a few research results have been achieved in this field, and rare results on bounded positive solutions in neutral delay difference equation with positive and negative coefficients. Reference studies the existence of positive solutions in second-order neutral delay difference equation with positive and negative coefficients $\Delta^{2}[x(n)+p x(n-\tau)]+R_{1}(n) x\left(n-\delta_{1}\right)-R_{2}(n) x\left(n-\delta_{2}\right)=o$, and gets a sufficient condition for the existence of positive solutions in this equation. Another reference promotes it to the higher order, that is, it focuses on following $l+1$ order difference equation with positive and negative coefficients $\Delta^{l+1}[x(n)+p x(n-\tau)]+R_{1}(n) x\left(n-\delta_{1}\right)-R_{2}(n) x\left(n-\delta_{2}\right)=o$

Where $l \in z^{+} ; p \in R ; \tau \in\{1,2, \cdots\} ; \delta_{1}, \delta_{2} \in\{0,1,2, \cdots\} ;\left\{R_{1}(n)\right\},\left\{R_{2}(n)\right\}$ are positive real sequences.

For convenience, now the basic concepts and marks in this paper are listed as follows:

“ $\Delta$ " Said for the forward difference operator $\Delta y(n)=y(n+1)-y(n) ; Z$ Said for the set composing by all integer; $R$ Said for the real number set. Set $a \in Z$, and $N(a)=\{a, a+1, \cdots\}, N=N(0),\{x(n)\}$ is named as the solution of difference equation (1) when the $\{x(n)\}$ satisfied the equation (1). When $n \in N(M)$, and $x(n)>0,\{x(n)\}$ is eventually positive solution which means that positive integral $M$ exists. When positive integral $M$ existed, where $n \in N(M)$, and $x(n)<0,\{x(n)\}$ is eventually negative solution. Oscillation refers to that $\{x(n)\}$ are neither eventually positive nor eventually negative, otherwise it called as nonoscillation.

Lemma 1 Assume

(i) $\sum_{n=1}^{+\infty} n^{l} R_{i}(n)<+\infty, i=1,2, n \in N\left(n_{0}\right)$ 
(ii) There exists a large enough positive integer $T_{1}$, to every $a>0$ and $n>\mathrm{T}_{1}$ hold, where $a R_{1}(n)-R_{2}(n) \geq 0$ (3)

(iii) $p \neq \pm 1$

hold, then Equation (1) has an eventually positive solution.

The proofing processes could be found in references.

\section{Main results and proof}

Among the sufficient conditions for the existence of nonoscillatory solution in differential equation(1), the condition (3) may look too restrictive; we will delete the strong condition (3), permit $p=1$, to get the overall sufficient condition for existence of eventually positive solution on the $p$ value in equation (1).

Theorem 1 Consider the difference equation with positive and negative coefficients

$\Delta^{l+1}[x(n)+p x(n-\tau)]+R_{1}(n) x\left(n-\delta_{1}\right)-R_{2}(n) x\left(n-\delta_{2}\right)=o$

Where $l \in z^{+} ; p \in R ; \tau \in N(1) ; \delta_{1}, \delta_{2} \in N ; R_{1}(n), R_{2}(n) \in C\left(\left[n_{0}, \infty\right), R^{+}\right)$

If condition (2) is hold, where $p \neq-1$, then equation (1) has an eventually positive solution.

Proof: Let the $B_{N}$ be the Banach space which is composed of all bounded real sequences $x=x(n)$ in $N\left(n_{0}\right)$, define sup norm $\|x\|=\sup x(n)$. The proof of Theorem 1 will be divided into five claims.

Claim 1. $p=1$

From condition (2), choose a large enough positive integer $N>\left(\mathrm{n}_{0}\right)$, so that when $n>\mathrm{N}$,

$\sum_{k=n}^{+\infty} k^{l} R_{1}(k) \leq \frac{1}{l+2}$

$\sum_{k=n}^{+\infty} k^{l} R_{2}(k) \leq \frac{1}{l+2}$

hold. Define a subset as $A=\left\{x \in B_{N}: l \leq x(n) \leq l+2, n \in N\left(n_{0}\right)\right\}$, then it is easy to see $A$ is a bounded, closed, and convex subset of $B_{N}$.

Define a mapping $T: A \rightarrow B_{N}$ as follows

$T x(n)=\left\{\begin{array}{cr}(l+1)+\sum_{i=1}^{+\infty} \sum_{j_{l-2}=n+(l i-1) \tau}^{n+l i \tau-1} \sum_{j_{l-3}=j_{l-2}}^{+\infty} \ldots \sum_{j_{1}=j_{2}}^{+\infty} \sum_{k=j_{1}}^{+\infty} \sum_{s=k}^{+\infty}(s-k+1) . \\ {\left[R_{1}(s) x\left(s-\delta_{1}\right)-R_{2}(s) x\left(s-\delta_{2}\right)\right]} & n \geq N \\ T x(N) & n_{0} \leq n<N\end{array}\right.$

Clearly, $T x$ is continuous. Next we will prove that $T$ is a self-mapping in $A$. When $n \geq N, \forall x \in A$, by using (5)and (7), we have

$$
\begin{aligned}
& T x(n) \leq(l+1)+\sum_{i=1}^{+\infty}\left[\sum_{j_{l-2}=n+(l i-1) \tau}^{n+l i z-1}+\sum_{j_{l-2}=n+(l i-l) \tau}^{n+(l i-1) \tau-1}\right] \sum_{j_{l-3}=j_{l-2}}^{+\infty} \cdots \sum_{j_{1}=j_{2}}^{+\infty} \sum_{k=j_{1}}^{+\infty} \sum_{s=k}^{+\infty}(s-k+1) R_{1}(s) x\left(s-\delta_{1}\right) \leq \\
& (l+1)+(l+2) \sum_{i=1}^{+\infty} \sum_{j_{l-2}=n+(l i-l) \tau}^{n+l i \tau-1} \sum_{j_{l-3}=j_{l-2}}^{+\infty} \cdots \sum_{j_{1}=j_{2}}^{+\infty} \sum_{k=j_{1}}^{+\infty} \sum_{s=k}^{+\infty}(s-k+1) R_{1}(s)= \\
& (l+1)+(l+2) \sum_{j_{l-2}=n}^{+\infty} \sum_{j_{l-3}=j_{l-2}}^{+\infty} \ldots \sum_{j_{1}=j_{2}}^{+\infty} \sum_{k=j_{1}}^{+\infty} \sum_{s=k}^{+\infty}(s-k+1) R_{1}(s) \leq \\
& (l+1)+(l+2) \sum_{s=n}^{+\infty} s^{l} R_{1}(s) \leq(l+1)+(l+2) \times \frac{1}{l+2}=l+2
\end{aligned}
$$


Furthermore, in view of (6) and (7), we have:

$T x(n) \geq(l+1)-(l+2) \sum_{s=n}^{+\infty} s^{l} R_{2}(s) \geq(l+1)-(l+2) \times \frac{1}{l+2}=l$

Clearly, when $n_{0} \leq n<N$, we have $l \leq x(n) \leq l+2$, thus $T x \in A$ which means that $T$ is the self- mapping in A.

Next we will prove that $T$ is a contraction mapping in $A$. When $n>\mathrm{N}$, for every $x_{1}, x_{2} \in A$, we have:

$\left|\operatorname{Tx}_{1}(n)-T x_{2}(n)\right| \leq \mid \sum_{i=1}^{+\infty} \sum_{j_{l-2}=n+(l i-1) \tau}^{n+l i \tau-1} \sum_{j_{l-3}=j_{l-2}}^{+\infty} \cdots \sum_{j_{1}=j_{2}}^{+\infty} \sum_{k=j_{1}}^{+\infty} \sum_{s=k}^{+\infty}(s-k+1)$.

$R_{1}(s)\left[x_{1}\left(s-\delta_{1}\right)-x_{2}\left(s-\delta_{2}\right)\right] \mid \leq$

$\sum_{j_{l-2}=n}^{+\infty} \sum_{j_{l-3}=j_{l-2}}^{+\infty} \cdots \sum_{j_{1}=j_{2}}^{+\infty} \sum_{k=j_{1}}^{+\infty} \sum_{s=k}^{+\infty}(s-k+1) R_{1}(s)\left\|x_{1}-x_{2}\right\| \leq$

$\sum_{s=n}^{+\infty} s^{l} R_{1}(s)\left\|x_{1}-x_{2}\right\| \leq \frac{1}{l+2}\left\|x_{1}-x_{2}\right\|$

Where $q=\frac{1}{l+2}$, so that $\left|T x_{1}(n)-T x_{2}(n)\right| \leq q\left\|x_{1}-x_{2}\right\|$.

Clearly, when $n_{0} \leq n<N$, we have $\left|T_{1}(n)-T x_{2}(n)\right| \leq q\left\|x_{1}-x_{2}\right\|$. Thus $T$ is a contraction mapping in $A$.

Above all, according to Banach contraction mapping, $T$ has a fixed point on $A$ such that $T x=x$. And $x=\{x(n)\}$ satisfies equation (7), so we have

$$
\begin{aligned}
& x(n)+x(n-\tau)=(2 l+4)+\sum_{i=1}^{+\infty} \sum_{j_{l-2}=n+(l i-l) \tau}^{n+l i \tau-1} \sum_{j_{l-3}=j_{l-2}}^{+\infty} \ldots \sum_{j_{1}=j_{2}}^{+\infty} \sum_{k=j_{1}}^{+\infty} \sum_{s=k}^{+\infty}(s-k+1) . \\
& {\left[R_{1}(s) x\left(s-\delta_{1}\right)-R_{2}(s) x\left(s-\delta_{2}\right)\right]=} \\
& (2 l+4)+\sum_{j_{l-2}=n}^{+\infty} \sum_{j_{l-3}=j_{l-2}}^{+\infty} \ldots \sum_{j_{1}=j_{2}}^{+\infty} \sum_{k=j_{1}}^{+\infty} \sum_{s=k}^{+\infty}(s-k+1)\left[R_{1}(s) x\left(s-\delta_{1}\right)-R_{2}(s) x\left(s-\delta_{2}\right)\right]
\end{aligned}
$$

To get the $l+1$ order difference equation from the above equation,

$\Delta^{l+1}[x(n)+x(n-\tau)]+R_{1}(n) x\left(n-\delta_{1}\right)-R_{2}(n) x\left(n-\delta_{2}\right)=o$

Thus, the fixed point $\{x(n)\}$ is a positive solution of equation (1). This completes the proof of Claim1.

Claim 2. $0 \leq p<1$

From condition (2), choose a sufficiently large positive integer $n_{1} \geq \max \left\{T_{1}, n_{0}+\delta\right\}$, where $\delta=\max \left\{\tau, \delta_{1}, \delta_{2}\right\}$, so that

$$
\begin{gathered}
\sum_{n=n_{1}}^{+\infty} n^{l} R_{1}(n) \leq \frac{M_{2}-(1-p)}{M_{2}} \\
\sum_{n=n_{1}}^{+\infty} n^{l} R_{2}(n) \leq \frac{1-p-p M_{2}-M_{1}}{M_{2}} \\
\sum_{n=n_{1}}^{+\infty} n^{l}\left[R_{1}(n)+R_{2}(n)\right]<1-p
\end{gathered}
$$

hold. Where $M_{1}$ and $M_{2}$ are positive constant, $1-M_{2}<p \leq \frac{1-M_{1}}{1+M_{2}}$.

Let $A=\left\{x \in B_{N}: M_{1} \leq x(n) \leq M_{2}, n \in N\left(n_{0}\right)\right\}$, then it is easy to see $A$ is a bounded, closed, and convex subset of $B_{N}$. 
Define a mapping $T: A \rightarrow B_{N}$ as follows

$$
T x(n)=\left\{\begin{array}{cc}
1-p-p x(n-\tau)+\sum_{s=n}^{+\infty} C_{s+l-n}^{l}\left[R_{1}(s) x\left(s-\delta_{1}\right)-\right. \\
\left.R_{2}(s) x\left(s-\delta_{2}\right)\right] & n \geq n_{1} \\
T x\left(n_{1}\right) & n_{0} \leq n<n_{1}
\end{array}\right.
$$

Clearly, $T x$ is continuous. Next we will prove that $T$ is a self- mapping in $A$.

When $n \geq n_{1}$, and $\forall x \in A$, by using (8) and (11), we have

$T x(n) \leq 1-p+\sum_{s=n}^{+\infty} C_{s+l-n}^{l} R_{1}(s) x\left(s-\delta_{1}\right) \leq$

$1-p+M_{2} \sum_{s=n}^{+\infty} s^{l} R_{1}(s) \leq 1-p+\frac{M_{2}\left(M_{2}-(1-p)\right)}{M_{2}}=M_{2}$

Furthermore, in view of (9) and (11), we have:

$\operatorname{Tx}(n) \geq 1-p-p x(n-\tau)-\sum_{s=n}^{+\infty} C_{s+l-n}^{l} R_{2}(s) x\left(s-\delta_{2}\right) \geq$

$1-p-p M_{2}-M_{2} \sum_{s=n}^{+\infty} s^{l} R_{2}(s) \geq 1-p-p M_{2}-\frac{M_{2}\left(1-p-p M_{2}-M_{1}\right)}{M_{2}}=M_{1}$

Clearly, when $n_{0} \leq n<n_{1}$, we have $M_{1} \leq x(n) \leq M_{2}$. Thus $T x \in A$ which means that $T$ is the self- mapping in A.

Next we will prove that $T$ is a contraction mapping in $A$. When $n>n_{1}$, for every $x_{1}, x_{2} \in A$, we have:

$\left|T x_{1}(n)-T x_{2}(n)\right| \leq\left|-p x_{1}(n-\tau)+p x_{2}(n-\tau)\right|+$

$\sum_{s=n}^{+\infty} C_{s+l-n}^{l} R_{1}(s)\left|x_{1}\left(s-\delta_{1}\right)-x_{2}\left(s-\delta_{1}\right)\right|+$

$\sum_{s=n}^{+\infty} C_{s+l-n}^{l} R_{2}(s)\left|x_{1}\left(s-\delta_{2}\right)-x_{2}\left(s-\delta_{2}\right)\right| \leq$

$p\left\|x_{1}-x_{2}\right\|+\left\|x_{1}-x_{2}\right\| \sum_{s=n}^{+\infty} s^{l}\left(R_{1}(s)+R_{2}(s)\right)=$

$\left\|x_{1}-x_{2}\right\|\left[p+\sum_{s=n}^{+\infty} s^{l}\left(R_{1}(s)+R_{2}(s)\right)\right]$

From condition (10), where $0<q_{1}<1$, so that $\left|T x_{1}(n)-T x_{2}(n)\right| \leq q_{1}\left\|x_{1}-x_{2}\right\|$.

Clearly, when $n_{0} \leq n<n_{1}$, we have $\left|T x_{1}(n)-T x_{2}(n)\right| \leq q\left\|x_{1}-x_{2}\right\|$. Thus $T$ is the contraction mapping in A.

According to Banach contraction mapping, $T$ has a fixed point on $A$ such that $T x=x$. And $x=\{x(n)\}$ we have

$T x(n)=\left\{\begin{array}{cc}1-p-p x(n-\tau)+\sum_{s=n}^{+\infty} C_{s+l-n}^{l}\left[R_{1}(s) x\left(s-\delta_{1}\right)-\right. \\ \left.R_{2}(s) x\left(s-\delta_{2}\right)\right] & n \geq n_{1} \\ T x\left(n_{1}\right) & n_{0} \leq n<n_{1}\end{array}\right.$

So this fixed point $x=\{x(n)\}$ is a positive sequence. 
To get the $l+1$ order difference equation from the above equation,

$\Delta^{l+1}[x(n)+p x(n-\tau)]+R_{1}(n) x\left(n-\delta_{1}\right)-R_{2}(n) x\left(n-\delta_{2}\right)=o$

Thus, the fixed point $\{x(n)\}$ is a positive solution of equation (1). This completes the proof of Claim2.

Claim 3. $p>1$

From condition (2), choose a large enough positive integer $n_{2}>\mathrm{T}_{1}>n_{0}$, where $n_{2}+\tau=n_{0}+\max \left\{\delta_{1}, \delta_{2}\right\}$, so that

$$
\begin{aligned}
& \sum_{n=n_{2}}^{+\infty} n^{l} R_{1}(n) \leq \frac{p\left(M_{4}-1\right)+1}{M_{4}} \\
& \sum_{n=n_{2}}^{+\infty} n^{l} R_{2}(n) \leq \frac{p\left(1-M_{3}\right)-\left(1+M_{4}\right)}{M_{4}} \\
& \sum_{n=n_{2}}^{+\infty} n^{l}\left[R_{1}(n)+R_{2}(n)\right]<p-1
\end{aligned}
$$

hold. Where $M_{3}$ and $M_{4}$ are positive constants, $\left(1-M_{3}\right) p \geq 1+M_{4}$ and $p\left(1-M_{4}\right)<1$

Let $A=\left\{x \in B_{N}: M_{3} \leq x(n) \leq M_{4}, n \in N\left(n_{0}\right)\right\}$, then it is easy to see $A$ is a bounded, closed, and convex subset of $B_{N}$.

Define a mapping $T: A \rightarrow B_{N}$ as follows

$$
T x(n)=\left\{\begin{array}{cc}
1-\frac{1}{p}-\frac{1}{p} x(n-\tau)+\frac{1}{p} \sum_{s=n+\tau}^{+\infty} C_{s+l-n-\tau}^{l}\left[R_{1}(s) x\left(s-\delta_{1}\right)-\right. \\
\left.R_{2}(s) x\left(s-\delta_{2}\right)\right] & n \geq n_{2} \\
T x\left(n_{2}\right) & n_{0} \leq n<n_{2}
\end{array}\right.
$$

Claim 4. $-1<p<0$

From condition (2), choose a large enough positive integer $n_{3} \geq \max \left\{T_{1}, n_{0}+\delta\right\}$, where $\delta=\max \left\{\tau, \delta_{1}, \delta_{2}\right\}$, so that

$$
\begin{aligned}
& \sum_{n=n_{3}}^{+\infty} n^{l} R_{1}(n) \leq \frac{M_{6}(1+p)-(1+p)}{M_{6}} \\
& \sum_{n=n_{3}}^{+\infty} n^{l} R_{2}(n) \leq \frac{(1+p)-M_{5}(1+p)}{M_{6}} \\
& \sum_{n=n_{3}}^{+\infty} n^{l}\left[R_{1}(n)+R_{2}(n)\right]<1+p
\end{aligned}
$$

hold. Where $M_{5}, M_{6}$ are positive constants, and $0<M_{5} \leq 1<M_{6}$.

Let $A=\left\{x \in B_{N}: M_{5} \leq x(n) \leq M_{6}, n \in N\left(n_{0}\right)\right\}$, then it is easy to see $A$ is a bounded, closed, and convex subset of $B_{N}$.

Define a mapping $T: A \rightarrow B_{N}$ as follows

$$
T x(n)=\left\{\begin{array}{cc}
1+p-p x(n-\tau)+\sum_{s=n}^{+\infty} C_{s+l-n}^{l}\left[R_{1}(s) x\left(s-\delta_{1}\right)-\right. \\
\left.R_{2}(s) x\left(s-\delta_{2}\right)\right] & n \geq n_{3} \\
T x\left(n_{3}\right) & n_{0} \leq n<n_{3}
\end{array}\right.
$$


Claim 5. $p<-1$

From condition (2), choose a large enough positive integer $n_{4}>\mathrm{T}_{1}>n_{0}$, where $n_{4}+\tau=n_{0}+\max \left\{\delta_{1}, \delta_{2}\right\}$, so that

$\sum_{n=n_{4}}^{+\infty} n^{l} R_{1}(n) \leq \frac{\left(M_{7}-1\right)(p+1)}{M_{8}}$

$\sum_{n=n_{4}}^{+\infty} n^{l} R_{2}(n) \leq \frac{\left(1-M_{8}\right)(1+p)}{M_{8}}$

$\sum_{n=n_{4}}^{+\infty} n^{l}\left[R_{1}(n)+R_{2}(n)\right]<-1-p$

hold. Where $M_{7}, M_{8}$ are positive constants, and $0<M_{7}<1<M_{8}$.

Let $A=\left\{x \in B_{N}: M_{7} \leq x(n) \leq M_{8}, n \in N\left(n_{0}\right)\right\}$, then it is easy to see $A$ is a bounded, closed, and convex subset of $B_{N}$.

Define a mapping $T: A \rightarrow B_{N}$ as follows

$$
T x(n)=\left\{\begin{array}{cc}
1+\frac{1}{p}-\frac{1}{p} x(n-\tau)+\frac{1}{p} \sum_{s=n+\tau}^{+\infty} C_{s+l-n-\tau}^{l}\left[R_{1}(s) x\left(s-\delta_{1}\right)-\right. \\
\left.R_{2}(s) x\left(s-\delta_{2}\right)\right] & n \geq n_{4} \\
T x\left(n_{4}\right) & n_{0} \leq n<n_{4}
\end{array}\right.
$$

Due to the proofs of Claim 3, 4, 5 are similar as Claim 2, these proofs are left out.

\section{References}

Cheng,Jinfa \& Z.Annie.Existence of Nonoscillatory Solution to Second Order Linear Neutral Delay Equation.System Science and Mathematics,2004,24(3)389-397.

Liu,Yue-hua \& Chen,Ya-bo.Existence of Nonoscillatory Solution of Second Order Linear Neutral Difference Equation ..Journal of Hunan Agricultural University(Natural Sciences),2001,27(1):76-79.

Lin,Wenxian.Neutral Partial Functional Differential Equation. Journal of Anhui University (Natural Science Edition), 2006,30(4):12-14.

Liang,Jingcui, Zhong,Xiaozhu,Wang,Donghua \& Ge,Lixia.Existence of Nonoscillatory Solution of Third Order Linear Neutral Delay Difference Equation. Journal of Yanshan University, 2006, 30(2):101-104.

Sun,Shu-rong \& Asymptptic.Behavior and Existence of Positive Solution for the Higher Order Neutral Difference Equations.Journal of Engineering Mathematics, 2002,19(3):15-20.

Xing,Hailong \& Zhong,Xiaozhu. Existence of Nonoscillatory Solution of High Order Linear Neutral Delay Difference Equation with Positive and Negative Coefficients. Journal of Yanshan University,2007,31(1):33-40. 